Draft Version OCtober 20, 2016

Preprint typeset using IATEX style AASTeX6 v. 1.0

\title{
ON THE DETERMINATION OF TRANSITING PLANET PROPERTIES FROM LIGHT AND RADIAL VELOCITY CURVES
}

\author{
John SouthworTh \\ Astrophysics Group, Keele University, Staffordshire, ST5 5BG, UK
}

\begin{abstract}
A recent publication has suggested a method to determine the masses and radii of the components of an eclipsing system using only a light curve and radial velocities of one component. If true, this would have immediate impact in expediting the study of transiting extrasolar planet and brown dwarf systems. The method is intended for situations where the mass ratio is significantly different from zero, but implicitly also requires the assumption that the mass ratio is negligible. We investigate both cases, finding that when the mass ratio is significant the method is mathematically identical to existing approaches, and when the mass ratio is negligible the equations become undefined. We therefore conclude that the method cannot be used to measure the physical properties of such systems from observations alone.
\end{abstract}

Keywords: planetary systems — stars: fundamental parameters

\section{INTRODUCTION}

Eclipsing binary stars for which radial velocities can be measured for both components are vital objects in stellar astrophysics, as the masses and radii of the components can be determined using only quantities directly measurable from light and velocity curves (Stebbins 1911; Andersen 1991; Torres et al. 2010). For eclipsing systems for which radial velocities can only be measured for one component (typically defined to be the primary component), this is not possible because one no longer has access to one of the quantities measured from observations (specifically the velocity amplitude of the secondary component). Examples of such eclipsing systems include low-mass eclipsing binaries (e.g. Fernandez et al. 2009), transiting brown dwarfs systems (e.g. Anderson et al. 2011) and transiting planetary systems (e.g. Torres et al. 2008).

Transiting planets have been the subject of extensive work in recent years, and several methods have been developed to provide the additional constraint required due to our inability to measure their physical properties directly. These include using predictions from theoretical stellar evolutionary models to constrain the properties of the host star (Cody \& Sasselov 2002; Sozzetti et al. 2007; Southworth 2009), applying an empirical mass-radius relation to the host star (Seager \& Mallén-Ornelas 2003; Southworth 2009; Enoch et al. 2010; Southworth 2011), characterising the host star using asteroseismology (e.g. Silva Aguirre et al. 2015) and measuring Doppler boosting and ellipsoidal variations (e.g. Faigler et al. 2013;
Esteves et al. 2013).

Montet et al. (2015, hereafter M15) recently presented a thorough and comprehensive study of the transiting brown dwarf system LHS 6343, originally discovered using photometric data from the Kepler satellite (Johnson et al. 2011). LHS 6343 contains a $62 \mathrm{M}_{\text {Jup }}$ brown dwarf orbiting a $0.36 \mathrm{M}_{\odot}$ M-dwarf every $12.7 \mathrm{~d}$. M15 presented a new method to determine the physical properties (specifically the masses and radii) of the two components of the system, which uses only quantities directly measurable from the light curve and radial velocities of the primary component. M15 found this method to work for systems where the mass ratio is not negligible and to allow the additional constraint to be bypassed. On closer inspection, the method requires two assumptions which are in mutual conflict: that the mass ratio be negligible and that the mass ratio be significantly different from zero. It is the purpose of the current work to investigate the method and determine the types of systems to which it may be applicable.

\section{RETRACING THE METHOD}

\subsection{Basic equations}

In this work we consider two spherical bodies which are orbiting each other. Kepler's Third Law can be written as:

$$
P^{2}=\frac{4 \pi^{2} a^{3}}{G\left(M_{1}+M_{2}\right)}
$$

where $P$ is orbital period, $a$ is semimajor axis, $G$ is the Newtonian gravitational constant, and $M_{1}$ and $M_{2}$ are 
the masses of the two bodies. By convention we expect $M_{1}>M_{2}$, and for extrasolar planets we can also write $M_{1} \gg M_{2}$.

The definition for mean density of a body is:

$$
\rho=\frac{\text { mass }}{\text { volume }}=\frac{3 M}{4 \pi R^{3}}
$$

where $R$ is the radius of the body in question.

Combining Equations 1 and 2 leads to an important result:

$$
\rho_{1}=\frac{3 \pi}{G P^{2}} \frac{a^{3}}{R_{1}^{3}} \frac{M_{1}}{M_{1}+M_{2}}=\frac{3 \pi}{G P^{2}}\left(\frac{a}{R_{1}}\right)^{3} \frac{1}{1+q}
$$

where $q=\frac{M_{2}}{M_{1}}$ is the mass ratio. This equation shows that the density of the primary object can be obtained as a function of a few physical constants $(G$ and $\pi)$, parameters determined from the light curve $\left(P\right.$ and $\frac{a}{R_{1}}$; see Russell 1912 and Seager \& Mallén-Ornelas 2003) and $q$. In the case of transiting planets one can further simplify this by assuming $q \approx 0$, in which case $\rho_{1}$ can be obtained purely from observed quantities:

$$
\rho_{1} \approx \frac{3 \pi}{G P^{2}}\left(\frac{a}{R_{1}}\right)^{3}
$$

An alternative approach to this approximation can be obtained by substituting the density of the secondary object into Equation 3 to find:

$$
\rho_{1}+k^{3} \rho_{2}=\frac{3 \pi}{G P^{2}}\left(\frac{a}{R_{1}}\right)^{3}
$$

where $k=\frac{R_{2}}{R_{1}}$ is the ratio of the radii of the two object. In the case of transiting planets one can apply the approximation that $R_{2}{ }^{3} \ll R_{1}{ }^{3}$ and thus $k^{3} \approx 0$, in which case Equation 5 reduces to Equation 4 above (see Winn 2010).

\subsection{The method introduced by $M 15$}

M15 defined two parameters, $c_{1}$ and $c_{2}$, which can be combined to determine the masses of the two bodies. The quantity $c_{1}$ comes from Equation 3 above and is:

$$
c_{1} \equiv 1+q=\left(\frac{3 \pi}{G P^{2}}\right)\left(\frac{1}{\rho_{1}}\right)\left(\frac{a}{R_{1}}\right)^{3}
$$

The parameter $c_{2}$ is very closely related to the mass function:

$$
f\left(M_{1}, M_{2}\right)=\frac{K_{1}^{3} P\left(1-e^{2}\right)^{3 / 2}}{2 \pi G}=\frac{M_{2}^{3} \sin ^{3} i}{\left(M_{1}+M_{2}\right)^{2}}
$$

(e.g. Hilditch 2001; Roy 2005) where $K$ is the velocity amplitude of the primary object and $e$ is the orbital eccentricity. The definition of $c_{2}$ by M15 is:

$$
c_{2} \equiv \frac{M_{2}^{3}}{\left(M_{1}+M_{2}\right)^{2}}=\frac{K_{1}^{3} P}{2 \pi G}\left(\frac{\sqrt{1-e^{2}}}{\sin i}\right)^{3}
$$

$c_{2}$ and $f\left(M_{1}, M_{2}\right)$ are practically equivalent for the systems we are considering here, because the high orbital inclinations required for transits to occur mean that $\sin i \approx 1$. The orbital inclination is also normally obtained to good precision from modelling the transit light curve, so the quantity $\sin i$ is precisely known.

From Equations 6 and 8 it follows that the masses of the two objects can be expressed as:

$$
\begin{aligned}
& M_{1}=\frac{c_{1}^{2} c_{2}}{\left(c_{1}-1\right)^{3}} \\
& M_{2}=\frac{c_{1}^{2} c_{2}}{\left(c_{1}-1\right)^{2}}
\end{aligned}
$$

From these quantities and the measured $\rho_{1}$ and $k$, the full physical properties of the system can be determined.

\subsection{Can this method be applied?}

In applying this approach to the analysis of a typical transiting planet or brown dwarf system, one would calculate $c_{1}$ and $c_{2}$ and then $M_{1}$ and $M_{2}$. The equation for $c_{1}$ (Equation 6) includes the density of the primary object. This can be determined from the light curve alone, but only under the assumption that $q \approx 0$ and therefore that Equation 4 is applicable. One then finds:

$$
c_{1}=\left(\frac{3 \pi}{G P^{2}}\right)\left(\frac{G P^{2} R_{1}^{3}}{3 \pi a^{3}}\right)\left(\frac{a}{R_{1}}\right)^{3}=1
$$

When applying this result to Equations 9 and 10, we find that $\left(c_{1}-1\right)=0$ and therefore the masses of the two components are undefined.

It is clear that the method breaks down once one adopts the approximation that $q \approx 0$. The method is therefore only useful if a measured value for $q$ is available, in which case one could use standard equations to achieve the same results. The method is therefore valuable only in that it provides a convenient way to determine the masses of the objects from observations and a known mass ratio; it cannot provide the masses from only observable quantities.

\section{DISCUSSION}

A method was recently presented for measuring the masses and radii of a transiting brown dwarf system based purely on parameters measured directly from a transit light curve and radial velocities of the host star. We have investigated this method and found that the situation divides into two regimes.

Mass ratio is negligible. This assumption is fundamental to the method proposed by M15, as it allows an approximation for the stellar density to be used and therefore the system of equations to be established. However, the assumption of a negligible mass ratio also leads to singularities in the equations which render them unusuable. We therefore refute the claim that "if the 
2

3

4
Doppler semiamplitude is known, the stellar mass can be measured exactly" (M15, Appendix, paragraph 2).

Mass ratio is not negligible. M15 do identify an issue with the equations becoming undetermined at low mass ratios, and in other parts of their work (e.g. Appendix, paragraph 13) mention that the adoption of a mass-radius relation of the form $M_{1} \propto R^{x}$, where $x$ is a real number typically around 3.0, allows the properties of the system to be obtained. This is the same approach as originally outlined by Seager \& Mallén-Ornelas (2003) and used with slight modification by Southworth (2009). In this regime, the method of equations is equivalent to most existing approaches used for determining the physical properties of transiting planetary systems, with all the same advantages and disadvantages of those approaches.

We therefore conclude that the method is only applicable in a subset of situations, in which case its perfor- mance is identical to many other mathematical prescriptions for measuring the properties of transiting planetary systems. An additional constraint beyond those obtained from modelling light and velocity curves is needed in all situations. A set of equations which make the impact of the additional constraint clear and quantifiable can be found in Southworth (2009, section 2.1).

\section{ACKNOWLEDGEMENTS}

JS acknowledges financial support from the Leverhulme Trust in the form of a Philip Leverhulme Prize. We thank Ben Montet for discussions. The following internet-based resources were used in research for this paper: the NASA Astrophysics Data System and the ar $\chi$ iv scientific paper preprint service operated by Cornell University.

\section{REFERENCES}

Andersen, J., 1991, A\&ARv, 3, 91

Anderson, D. R., et al., 2011, ApJ, 726, L19

Cody, A. M., Sasselov, D. D., 2002, ApJ, 569, 451

Enoch, B., Collier Cameron, A., Parley, N. R., Hebb, L., 2010, A\&A, 516, A33

Esteves, L. J., De Mooij, E. J. W., Jayawardhana, R., 2013, ApJ, 772,51

Faigler, S., Tal-Or, L., Mazeh, T., Latham, D. W., Buchhave, L. A., 2013, ApJ, 771, 26

Fernandez, J. M., et al., 2009, ApJ, 701, 764

Hilditch, R. W., 2001, An Introduction to Close Binary Stars,

Cambridge University Press, Cambridge, UK

Johnson, J. A., et al., 2011, ApJ, 730, 79

Montet, B. T., et al., 2015, ApJ, 800, 134

Roy, A. E., 2005, Orbital motion, 4th edition, Institute of

Physics Publishing, Bristol, UK
Russell, H. N., 1912, ApJ, 35, 315

Seager, S., Mallén-Ornelas, G., 2003, ApJ, 585, 1038

Silva Aguirre, V., et al., 2015, MNRAS, 452, 2127

Southworth, J., 2009, MNRAS, 394, 272

Southworth, J., 2011, MNRAS, 417, 2166

Sozzetti, A., Torres, G., Charbonneau, D., Latham, D. W., Holman, M. J., Winn, J. N., Laird, J. B., O'Donovan, F. T., 2007, ApJ, 664, 1190

Stebbins, J., 1911, ApJ, 34, 112

Torres, G., Winn, J. N., Holman, M. J., 2008, ApJ, 677, 1324

Torres, G., Andersen, J., Giménez, A., 2010, A\&ARv, 18, 67

Winn, J. N., 2010, Exoplanet transits and occultations, p. 55 